\title{
Effect of two oral formulations of cannabidiol on responses to emotional stimuli in healthy human volunteers: pharmaceutical vehicle matters
}

\author{
José A. Crippa, ${ }^{1,2}$ iD Luiz C. Pereira Junior, ${ }^{3}$ Lívia C. Pereira, ${ }^{1}$ Patrícia M. Zimmermann, ${ }^{4}$ Liberato
} Brum Junior, ${ }^{4}$ Letícia M. Rechia, ${ }^{4}$ Isabella Dias, ${ }^{1}$ Jaime E. Hallak, ${ }^{1,2}$ iD Alline C. Campos, ${ }^{1,5}$ iD Francisco S. Guimarães, ${ }^{1,5}$ (iD Regina H. Queiroz, ${ }^{2,3}$ (iD Antonio W. Zuardi ${ }^{1,2}$ (iD

${ }^{1}$ Departamento de Neurociências e Ciências do Comportamento, Faculdade de Medicina de Ribeirão Preto (FMRP), Universidade de São Paulo (USP), Ribeirão Preto, SP, Brazil. ${ }^{2}$ Instituto Nacional de Ciência e Tecnologia, Medicina Translacional (INCT-TM), Conselho Nacional de Desenvolvimento Científico e Tecnológico (CNPq), Ribeirão Preto, SP, Brazil. ${ }^{3}$ Departamento de Análises Clínicas, Toxicológicas e Bromatológicas, Faculdade de Ciências Farmacêuticas de Ribeirão Preto, USP, Ribeirão Preto, SP, Brazil. ${ }^{4}$ Prati-Donaduzzi, Toledo, PR, Brazil. ${ }^{5}$ Departamento de Farmacologia, FMRP, USP, Ribeirão Preto, SP, Brazil.

\begin{abstract}
Objective: To compare plasma concentrations of cannabidiol (CBD) following oral administration of two formulations of the drug (powder and dissolved in oil), and to evaluate the effects of these distinct formulations on responses to emotional stimuli in healthy human volunteers.

Methods: In a randomized, double-blind, placebo-controlled, parallel-group design, 45 healthy male volunteers were randomly assigned to three groups of 15 subjects that received either $150 \mathrm{mg}$ of CBD powder; $150 \mathrm{mg}$ of CBD dissolved in corn oil; or placebo. Blood samples were collected at different times after administration, and a facial emotion recognition task was completed after 150 min.

Results: There were no significant differences across groups in the subjective and physiological measures, nor in the facial emotion recognition task. However, groups that received the drug showed statistically significant differences in baseline measures of plasma CBD, with a significantly greater difference in favor of the oil formulation.

Conclusion: When administered as a single 150-mg dose, neither formulation of oral CBD altered responses to emotional stimuli in healthy subjects. The oil-based CBD formulation resulted in more rapid achievement of peak plasma level, with an approximate fourfold increase in oral bioavailability.
\end{abstract}

Keywords: Cannabidiol; CBD; drug interactions; pharmacokinetics

\section{Introduction}

Cannabidiol (CBD) is one of the main components of Cannabis sativa. It does not cause the typical effects of cannabis in humans, and has therapeutic potential for many clinical conditions. ${ }^{1}$ One of the greatest challenges of oral CBD administration is that this cannabinoid undergoes intense first-pass metabolism, as it is mostly metabolized by human liver microsomes (HLMs), the so-called CYP450 complex, and presents low solubility in aqueous solutions. ${ }^{2,3}$ Several studies in humans have shown beneficial effects of CBD in the treatment of different neuropsychiatric disorders, using oral administration of the drug dissolved in oil ${ }^{4,5}$ or in powder form. ${ }^{6,7}$ However, evidence on the plasma concentrations of CBD following oral administration with different vehicles is still scarce. ${ }^{8-10}$ Knowledge of the exact impact of standardized, oil-based formulations on CBD pharmacokinetics is

Correspondence: José A. Crippa, Departamento de Neurociências e Ciências do Comportamento, Faculdade de Medicina de Ribeirão Preto (FMRP), Universidade de São Paulo (USP), Av. Bandeirantes, 3900, $3^{\circ}$ andar, CEP 14049-900, Ribeirão Preto, SP, Brazil.

E-mail: jcrippa@fmrp.usp.br

Submitted Dec 03 2020, accepted Mar 02 2021, Epub May 282021. essential to predicting dosage requirements; assessing changes in dosage; estimating rates of elimination and rate of absorption; characterizing intra- and inter-subject variability; understanding concentration-effect relationships; and establishing safety margins, efficacy parameters, and the therapeutic window. In turn, understanding the pharmacokinetics of CBD is critical to comprehending the time to onset, intensity, and duration of its pharmacodynamic effects, thus maximizing therapeutic effects and minimizing adverse effects.

Therefore, given the increasing interest in the therapeutic uses of CBD, the primary objective of the present study is to compare plasma concentrations of CBD following oral administration of two formulations of the drug, in powder form and dissolved in oil, while controlling for possible acute side effects, in order to determine the most suitable form of CBD administration. Moreover, there is evidence that certain medications (especially

How to cite this article: Crippa JA, Pereira Junior LC, Pereira LC, Zimmermann PM, Brum Junior L, Rechia LM, et al. Effect of two oral formulations of cannabidiol on responses to emotional stimuli in healthy human volunteers: pharmaceutical vehicle matters. Braz J Psychiatry. 2022;44:15-20. http://dx.doi.org/10.1590/1516-4446-20201684 
anxiolytics) affect the processing of external social cues, which could result in biases in the attention to and interpretation of social threat. ${ }^{11}$ Tasks based on the recognition of facial emotions have, therefore, been widely used to assess biases in the evaluation of social judgment (positive or negative evaluations by others), including the effect of cannabinoid agents. ${ }^{12}$ Thus, we also evaluated the effects of these two formulations of CBD on responses to emotional stimuli in healthy human volunteers.

\section{Methods}

\section{Subjects}

Our estimation of sample size was based on prior studies that assessed the effects of CBD on anxiety produced by public speaking with the Visual Analog Mood Scale (VAMS). ${ }^{13,14}$ Considering the average result for the anxiety factor of the VAMS scale, we can expect a significant difference of $10.8 \mathrm{~mm}$ and a standard deviation of $13 \mathrm{~mm}$ for the placebo group and $8.5 \mathrm{~mm}$ for the CBD group. Assuming $80 \%$ test power, to detect a difference at the $5 \%$ significance level, the minimum number of volunteers was established as 15 per group.

The study included 45 healthy male volunteers, all nonsmokers, aged 18-35 years, who had not used cannabis over the previous month and with a lifetime history of no more than 15 episodes of exposure to cannabis; none had ever used any other illegal drug. The participants had not taken any medications for at least 3 months before the study. Exclusion criteria included using any medication during the study period and the presence of psychiatric disorders as assessed with the Structured Clinical Interview for DSM-IV (SCID-IV). Also, subjects were excluded if they had a history of clinically significant general medical conditions (cardiovascular, pulmonary, hepatic, renal, hematologic, gastrointestinal, endocrine, immunologic, dermatologic, neurologic, oncologic) or any other condition that could, in the opinion of the investigators, affect the safety or validity of the study results.

In a randomized, double-blind, placebo-controlled, parallel-group design, volunteers were randomly assigned to three groups of 15 subjects that received: 1 ) CBD in powder form, 2) CBD dissolved in corn oil, or 4) placebo. Groups were matched with respect to age, educational attainment (complete higher education), and body mass index (mean, $24 \pm 3 \mathrm{~kg} / \mathrm{m}^{2}$ ). To ensure the adequacy of the matching procedure, the treatment arm of the first participant was randomly drawn among the three treatment options available; the second participant (whose characteristics were matched to the first one's) had his treatment arm randomly drawn between the two remaining options; and the third participant (matched with the two previous ones) was assigned to the remaining arm. This procedure was repeated until all participants were assigned to one of the three groups.

\section{Procedure}

Each volunteer attended a single experimental session. CBD $(99.6 \%$ pure, with no other cannabinoids, provided by THC-Pharm, Frankfurt, Germany) was placed in identical gelatin capsules containing $150 \mathrm{mg}$ of powdered CBD, $150 \mathrm{mg}$ of CBD dissolved in corn oil, or cornstarch (placebo). The dose was chosen based on the minimum safe range observed in previous studies that detected an acute anxiolytic effect. ${ }^{1,4,8,14}$

Volunteers who agreed to participate were instructed to abstain from drugs and medications for 30 days and from alcohol and caffeine 24 hours before the experimental session. On the scheduled date, participants came to the laboratory after having a standardized breakfast $(200 \mathrm{~mL}$ of chocolate milk, two slices of plain bread and butter, and a medium-sized apple) around $30 \mathrm{~min}$ before receiving one of the CBD formulations or placebo. Administering CBD under such conditions allows for more precise assessment of pharmacokinetic parameters and is more representative of orally administered CBD products. A venous cannula was inserted on the left arm in righthanded volunteers and on the right arm in left-handed volunteers. The first blood sample and baseline measures were then collected. Immediately afterwards, the volunteers received a capsule containing the treatment they had been assigned to. Blood samples were collected through the punctured vein into heparin-containing tubes (Liquemine $^{\circledR}$ ) at $30,60,90,120,150,180$, and $240 \mathrm{~min}$ after administration of the capsules. Subjective and physiological measures were collected at the same time points. At 60 minutes after medication intake, a simulated public speaking test (SPST) was begun. A facial emotion recognition task was completed 150 min after capsule intake, in the same experimental session. The interval between capsule administration and the task measurements was chosen based on previous evidence of the time to peak plasma concentration of an oral dose of CBD. ${ }^{1,2,9}$

We decided to include only male subjects in the study, as it is now well recognized that human pharmacokinetic parameters of orally administered cannabinoids are altered by sex differences. ${ }^{15}$ The same occurs concerning the facial recognition test; females have shown enhanced sensitivity to anger and fear relative to males, among other inequalities. ${ }^{16}$

\section{Assessment instruments}

\section{Subjective assessment instruments}

The VAMS ${ }^{17}$ consists of 16 items, each of which is formed by two adjectives describing opposite subjective states (e.g., calm-excited), connected by a $100 \mathrm{~mm}$ line. Volunteers are asked to rate the way they feel in respect to each of the adjectives by making a vertical mark on the line, where the center indicates the way the person usually feels. Factor analysis has shown that the scale has four factors: mental sedation, physical sedation, anxiety, and other feelings and attitudes. ${ }^{18}$ The 16 VAMS items are grouped into the following four domains for analysis: 1) anxiety (items calm-excited, relaxed-tense, and tranquil-troubled); 2) sedation (items alert-drowsy and attentive-dreamy); 3) cognitive impairment (items quick witted-mentally slow, proficient-incompetent, energeticlethargic, clear headed-muzzy, gregarious-withdrawn, 
well-coordinated-clumsy, and strong-feeble); and 4) distress (items interested-bored, happy-sad, contenteddiscontented, and amicable-antagonistic). The individual VAMS domain scores comprise the sum of each factor item that was previously multiplied by each of its factor loadings.

\section{Physiological measures}

Physiological measures were collected during two-minute periods at different points of the procedure. Blood pressure (BP) and heart rate (HR; calculated through the interval between heart beats) were continually monitored and recorded through a multiparameter monitor (DX2022, Dixtal, Manaus, Brazil).

\section{Simulated public speaking test (SPST)}

The SPST was developed and validated by McNair et al. ${ }^{19}$ Detailed descriptions of the protocol are available elsewhere. ${ }^{13,18,20}$ Briefly, the subject is requested to prepare a speech and speak in front of a video camera. Before and after each procedure, the subjects complete the rating scales.

\section{Facial emotion recognition task}

In this study, we used a computerized task developed by Arrais et al. in $2010^{21}$ and based on the Pictures of Facial Affect series. ${ }^{22}$ The task used black-and-white pictures of two men and two women representing six basic emotions (happiness, fear, disgust, anger, sadness, and surprise) and an emotionally neutral expression. The stimuli were organized in 24 blocks ( 4 actors $\times 6$ emotions), in which emotional intensity increased gradually from a neutral face to a "full expression" in $10 \%$ increments. Participants were asked to label the emotion displayed as soon as they were able to recognize it. The emotional intensity of the stimuli was increased by the volunteer using a response box containing eight buttons with the labels of each emotion and "Advance" and "Enter" keys, respectively used to increase the emotional intensity of the stimuli and to start another block after a response was given. There were no time constraints on the task, and the blocks were randomly distributed for each participant. Before beginning the actual trial, participants completed two practice blocks and, at the end of the task, the software generated a report informing the gender of the actor depicted, the time of exhibition of each stimulus, the total time of the task, the participant's response, and the actual emotion displayed in each block.

\section{Bioanalytical method}

Analytical standards, reagents, blank plasma samples

The CBD standard was supplied by THC Pharm (Frankfurt, Germany). Ampules of $\Delta 9$-THC $(1 \mathrm{mg} / \mathrm{mL})$ and $\Delta 9$-THC-d3 (1 mg/mL), both dissolved in methanol, were purchased from Cerilliant Corporation (Round Rock, USA), and ampules of CBD (1 mg/mL) dissolved in methanol for quality control were kindly donated by
Prof. Marilyn A. Huestis, National Institute on Drug Abuse (Baltimore, USA). The derivatizing agent bis(trimethylsilyl)-trifluoroacetamide (BSTFA) with 1\% trimethylchlorosilane (TMC) was supplied by Sigma-Aldrich (St. Louis, USA), HPLC grade methanol was provided by J.T. Baker (Phillipsburg, USA), hexane was supplied by Mallinckrodt (Staines-upon-Thames, UK), and petroleum ether and acetonitrile were supplied by Merck (Darmstadt, Germany). Blank human plasma was provided from individuals not exposed to CBD or $\triangle 9$-THC from the Hospital São Francisco Blood Bank (Ribeirão Preto, Brazil).

\section{Instrumentation and analytical conditions}

Analysis was performed by gas-chromatography mass spectrometry (GC/MS) (Agilent, Varian Saturn 2000 model, Santa Clara, USA) using the ion trap technique. Separation of the cannabinoids was done in a capillary column HP-5MS (5\% phenyl-methylpolysiloxane, $30 \mathrm{~m} \mathrm{x}$ $0.25 \mathrm{~mm}$, film $0.25 \mu \mathrm{m}$; Agilent) with helium 5.0 (White Martins, Rio de Janeiro, Brazil) as the carrier gas at a flow rate of $1 \mathrm{~mL} / \mathrm{min}$. The initial temperature of the column was $80{ }^{\circ} \mathrm{C}(1 \mathrm{~min})$, followed by ramps of $30{ }^{\circ} \mathrm{C} / \mathrm{min}$ up to $250{ }^{\circ} \mathrm{C}$ and $10{ }^{\circ} \mathrm{C} / \mathrm{min}$ up to $300{ }^{\circ} \mathrm{C}$. The injector temperature was $260^{\circ} \mathrm{C}$, operating in splitless mode. The standards were initially injected in full scan detection mode. Next, the most abundant ions were selected for use in the single ion selected (SIS) detection mode.

Calibration standards (CS), quality controls (QC) and internal standard (IS)

CBD (1 $\mathrm{mg} / \mathrm{mL}$ ) was prepared in methanol (stock solution). From this stock solution, dilutions were made as follows: $100,200,500,1,000,2,000,2,500,3,000,4,000$, $5,000,6,000,8,000$, and $10,000 \mathrm{ng} / \mathrm{mL}$. For the $\mathrm{QC}$, solutions of $300,1,400$ and $3,600 \mathrm{ng}$ of $\mathrm{CBD} / \mathrm{mL}$ of methanol were prepared from an ampule of $1 \mathrm{~mL}$ (CBD $1 \mu \mathrm{g} / \mathrm{mL})$. For the deuterated IS, a solution of $1,000 \mathrm{ng}$ $\triangle 9-T H C-d 3 / m L$ of methanol was prepared from an ampule of $1 \mathrm{~mL}$ (Delta $9-\mathrm{THC}-\mathrm{d} 3,1 \mu \mathrm{g} / \mathrm{mL}$ ).

\section{Sample preparation for CS, QC and volunteers}

Aliquots $(50 \mu \mathrm{L})$ of $\mathrm{CBD}$ solutions at each concentration and one aliquot of IS were added to $0.5 \mathrm{~mL}$ of blank human plasma. After shaking in a vortex for $30 \mathrm{~s}, 2.5 \mathrm{~mL}$ of petroleum ether was added for extraction under agitation in a mechanical shaker for $30 \mathrm{~min}$, followed by centrifugation $\left(-10^{\circ} \mathrm{C}\right)$ at $1,800 \times \mathrm{g}$ for $5 \mathrm{~min}$. After centrifugation, $1 \mathrm{~mL}$ of the supernatant was removed and evaporated to dryness under nitrogen flow at $37^{\circ} \mathrm{C}$. The residue was re-dissolved in $50 \mu \mathrm{L}$ of acetonitrile and the tubes were vigorously vortexed for $30 \mathrm{~s}$. Then, $40 \mu \mathrm{L}$ of the extract was transferred to auto-sampler vials, and $20 \mu \mathrm{L}$ of bis(trimethylsilyl)-trifluoroacetamide (BSTFA) were added for derivatization at $80{ }^{\circ} \mathrm{C}$ for $45 \mathrm{~min}$. After cooling at room temperature, $1 \mu \mathrm{L}$ was injected into the gas chromatograph. ${ }^{23}$

Plasma $(0.5 \mathrm{~mL})$ from volunteers was added to $50 \mu \mathrm{L}$ of IS solution and vortexed for $30 \mathrm{~s}$. Samples were then prepared following the same procedure described above. 


\section{Statistical analysis}

The scores of the VAMS domains and the physiological measures were analyzed with repeated-measures analysis of variance (ANOVA) including the factors phase, group, and phase $\times$ group interaction. Performance in the facial emotion recognition task was assessed using multivariate ANOVA (MANOVA). Analyses were performed in SPSS version 17 and expressed as $F, p$-value, and partial eta squared $\left(\eta_{\mathrm{p}}^{2}\right)$. The level of statistical significance adopted was $p<0.05$.

The plasma concentration of $\mathrm{CBD}$ in the two groups that received the drugs were analyzed with repeatedmeasures ANOVA, including the factors phase, group, and phase $\times$ group interaction. When the phase $\times$ group interaction was significant, the test of within-subjects contrast was used to assess differences between groups in each measure concerning the baseline.

\section{Ethics statement}

The ethics committee of the Faculdade de Ciências Farmacêuticas de Ribeirão Preto, Universidade de São Paulo (USP) approved the study protocol (FCFRP 290-12). All volunteers gave written consent to participate after receiving detailed information about the experimental procedures.

\section{Results}

During the SPST, there were no significant differences across groups in the repeated-measures ANOVA (phase $\times$ group interaction) of the four VAMS domains (anxiety $\left[\mathrm{F}_{8,168}=0.579 ; p=0.79 ; \eta_{\mathrm{p}}^{2}=0.027\right]$; sedation $\left[\mathrm{F}_{8,168}=\right.$ $\left.0.894 p=0.52 ; \eta_{p}^{2}=0.041\right]$; cognitive impairment $\left[F_{8,168}=\right.$ $\left.1.518 ; p=0.15 ; \eta_{p}^{2}=0.067\right]$, and discomfort $\left[F_{8,168}=\right.$ 1.186; $\left.\left.p=0.31 \eta_{p}^{2}=0.053\right]\right)$, heart rate $\left(F_{10,205}=1.086\right.$; $\left.p=0.38 ; \eta_{p}^{2}=0.50\right)$, or systolic $\left(F_{10,205}=0.919 ; p=0.52\right.$ $\left.\eta_{p}^{2}=0.043\right)$ and diastolic $\left(F_{10,205}=1.184 ; p=0.30 ; \eta_{p}^{2}=\right.$ $0.055)$ blood pressure. Thus, the volunteers presented no sedation and no cognitive or physiological alterations at the different time points of data collection. No significant side effects were recorded in any of the groups assessed through verbal reports.

For the facial emotion recognition task, MANOVA showed no significant differences across groups $\left(F_{12,72}=\right.$ $1.68 ; p=0.094)$ in terms of emotional intensity for recognition, assessed at 150 minutes after the administration of $\mathrm{CBD}$.

The collection of blood samples at baseline proved important, as we could exclude the presence of any cannabinoids (including THC and CBD) in the blood of participants. According to the chromatographic analysis, the pharmacokinetic behavior of the two CBD formulations revealed a mean plasma peak level of $20.70 \pm$ $1.68 \mathrm{ng} / \mathrm{mL}$ for CBD in powder form 2.5 hours after intake, and of $82.59 \pm 9.52 \mathrm{ng} / \mathrm{mL}$ for CBD dissolved in oil after 2 hours. The plasma concentrations of CBD began to decrease after these intervals.

The formulation of CBD dissolved in corn oil displayed a kinetic behavior similar to the kinetic curve observed for the powder formulation, but with the peak plasma concentration reached in a shorter interval.

Concerning the plasma concentrations of $\mathrm{CBD}$, the repeated-measures ANOVA showed significant differences related to phase $\left(F_{7,168}=919.4 ; p<0.001 ; \eta_{p}^{2}=\right.$ $0.975)$, group $\left(F_{1,24}=1,616.4 ; p<0.001 ; \eta_{p}^{2}=0.985\right)$, and phase $\times$ group interaction $\left(F_{7,168}=464.8 ; p<\right.$ $\left.0.001 ; \eta_{\mathrm{p}}^{2}=0.951\right)$. Contrast analysis showed that, after 1 hour, all values differed from baseline for time $(p<$ $0.001)$ and time $\times$ group interaction $(p<0.001)$. The two groups (powder and oil) showed significant differences relative to the baseline measures, with a more substantial difference in the group that received the oil formulation (Figure 1).

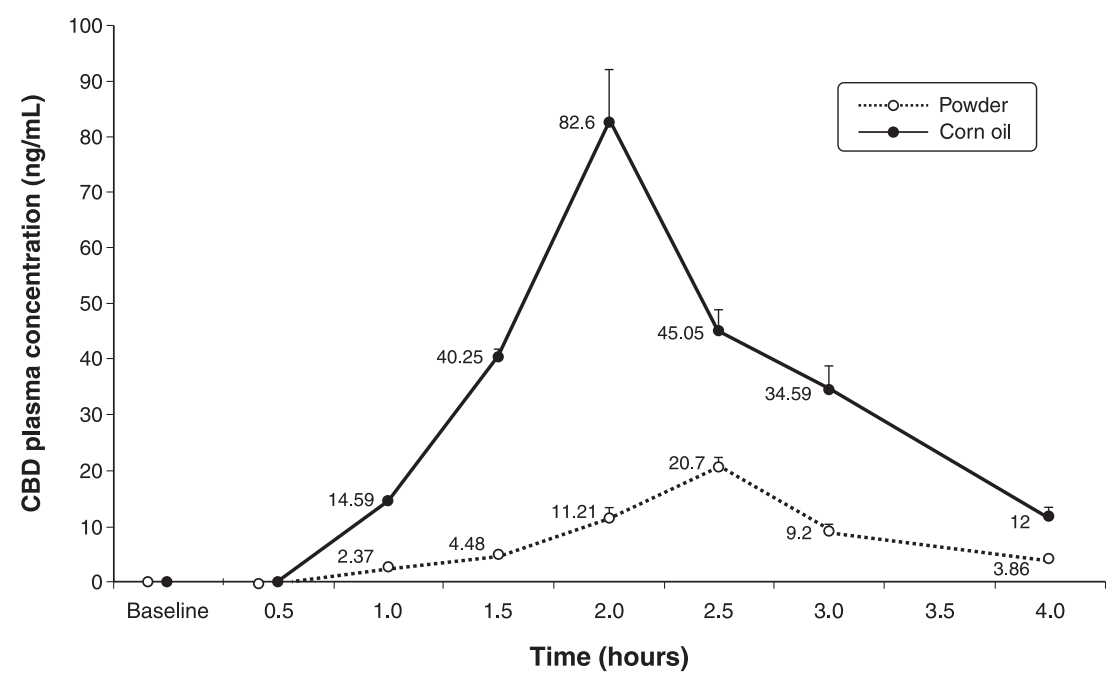

Figure 1 Comparison of mean plasma concentrations $(\mathrm{ng} / \mathrm{mL})$ of cannabidiol (CBD), administered in oil or powder form, over time. At a 150-mg oral dose, the plasma concentration of CBD over time was higher in the group of volunteers that received the corn oil formulation $(n=15)$ than in the group that took the powder formulation $(n=15)$. 


\section{Discussion}

We observed that, at a single, orally administered 150-mg dose, neither formulation (powder versus oil) of CBD altered responses to emotional stimuli in healthy subjects. This is in line with data from a recent human experiment in which single low doses of oral CBD did not affect responses to emotional stimuli, ${ }^{24}$ and highlights the inverted $U$-shaped profile of this cannabinoid in acute induced anxiety. ${ }^{20}$ However, it is essential to consider that the anxiogenic procedure of blood sample collection over time during the study protocol may have interfered with the subjective findings.

CBD dissolved in vegetable oil proved to be the most adequate dosage form to reach higher plasma levels of the drug. Between the two formulations used, CBD in oil resulted in a quicker peak plasma level compared to the powder formulation, with an approximate fourfold increase in oral bioavailability. This is consistent with the observation that CBD is poorly soluble in aqueous solutions and has a highly lipophilic profile, with increased solubility in oil and, hence, increased absorption and bioavailability, as reported for other drugs. ${ }^{25}$

Cell membranes are formed mainly of phospholipids, which contributes to a higher permeabilization of lipophilic substances when formulated in vehicles that have the same physicochemical profile. This vehicle may facilitate transit of the substance to the intracellular milieu because it diffuses through the plasma membrane (as both have the same profile), reducing absorption time and increasing the absorbed amount, which results in higher plasma concentrations. This is particularly relevant considering that the oral bioavailability of CBD in humans is low, at around $6 \%,{ }^{26,27}$ and bearing in mind that this has been the most widely used formulation for products consisting exclusively of CBD (such as Sativex ${ }^{\circledR}$ from GW-Pharm, for example). In addition to vegetable oil, CBD is also soluble in ethanol. ${ }^{24}$ Still, its hazardous long-term and potential additive, synergistic, and interactive effects when combined with different drugs limit ethanol as a solvent vehicle for oral CBD formulations. This highlights the importance of careful selection among different oral formulations of CBD for investigation of its therapeutic effects.

In the present study, the two oral formulations of CBD were well tolerated, and no participants reported any adverse effects. These findings agree with the commonly described favorable safety profile of CBD in humans. ${ }^{28}$ Since CBD has been shown to have therapeutic potential in several disorders, including epilepsy, posttraumatic stress disorder, and Parkinson's disease, it is essential to understand the actions of CBD in these conditions and its distinct profiles in these populations, as they may not be comparable to the action of CBD in healthy volunteers. ${ }^{1}$ Also, there is evidence suggesting that oral CBD should not be administered under fasting conditions to allow for optimal absorption in order to achieve successful therapeutic effects, as reported recently in pharmacokinetic studies. $^{29}$

Finally, future studies investigating dose-response patterns with different oral formulations of CBD are warranted, especially if we take into consideration evidence concerning the inverted U-shaped doseresponse curve of the substance, ${ }^{14,20,30}$ the imprecise therapeutic window of the drug for each medical condition, ${ }^{1}$ and the interactions between CBD and other drugs commonly prescribed in clinical practice. ${ }^{31,32}$

\section{Acknowledgements}

This study was funded by Instituto Nacional de Ciência e Tecnologia Translacional em Medicina (INCT-TM) Conselho Nacional de Desenvolvimento Científico e Tecnológico (CNPq) and by Fundação de Amparo à Pesquisa do Estado de São Paulo (FAPESP; 2008/09009-2). It was also supported by CNPq/MS/SCTIE/DECIT 26/2014 Pesquisas sobre Distúrbios Neuropsiquiátricos (466805/ 2014-4). JAC received a grant from the University Global Partnership Network (UGPN) - Global Priorities in Cannabinoid Research Excellence Program. JAC, JEH, FSG, and AWZ are recipients of CNPq research fellowships.

The authors thank Mrs. Sandra Bernardo for her technical support.

\section{Disclosure}

JAC is a member of the International Advisory Board of the Australian Centre for Cannabinoid Clinical and Research Excellence (ACRE) - National Health and Medical Research Council (NHMRC). JAC and JEH have received personal consultation fees and travel support to attend scientific meetings from BSPG-Pharm. JAC, JEH, $F S G$, and AWZ are coinventors of the patent "Fluorinated CBD compounds, compositions and uses thereof. Pub. No.: WO/2014/108899. International Application No.: PCT/ IL2014/050023," Def. US number Reg. 62193296; July 29, 2015; INPI on August 19, 2015 (BR1120150164927; Mechoulam R, Zuardi AW, Kapczinski F, Hallak JEC, Guimarâes FS, Crippa JAS, Breuer A). Universidade de São Paulo (USP) has licensed this patent to Phytecs Pharm (USP Resolution 15.1.130002.1.1) and has an agreement with Prati-Donaduzzi to "develop a pharmaceutical product containing synthetic CBD and prove its safety and therapeutic efficacy in the treatment of epilepsy, schizophrenia, Parkinson's disease, and anxiety disorders." JAC, JEH, FSG, and AWZ are coinventors of the patent "Cannabinoid-containing oral pharmaceutical composition, method for preparing and using same," INPI on September 16, 2016 (BR 112018005423-2).

\section{References}

1 Crippa JA, Guimarães FS, Campos AC, Zuardi AW. Translational investigation of the therapeutic potential of cannabidiol (CBD): toward a new age. Front Immunol. 2018;9:2009.

2 Harvey DJ, Samara E, Mechoulam R. Comparative metabolism of cannabidiol in dog, rat and man. Pharmacol Biochem Behav. 1991;40:523-32.

3 Jiang R, Yamaori S, Takeda S, Yamamoto I, Watanabe K. Identification of cytochrome P450 enzymes responsible for metabolism of cannabidiol by human liver microsomes. Life Sci. 2011;89:165-70.

4 Chagas MH, Zuardi AW, Tumas V, Pena-Pereira MA, Sobreira ET, Bergamaschi MM, et al. Effects of cannabidiol in the treatment of patients with Parkinson's disease: an exploratory double-blind trial. J Psychopharmacol. 2014;28:1088-98. 
5 Linares IM, Guimaraes FS, Eckeli A, Crippa AC, Zuardi AW, Souza JD, et al. No acute effects of cannabidiol on the sleep-wake cycle of healthy subjects: a randomized, double-blind, placebo-controlled, crossover study. Front Pharmacol. 2018;9:315.

6 Fusar-Poli P, Crippa JA, Bhattacharyya S, Borgwardt SJ, Allen P, Martin-Santos R, et al. Distinct effects of \{delta\}9-tetrahydrocannabinol and cannabidiol on neural activation during emotional processing. Arch Gen Psychiatry. 2009;66:95-105.

7 Bhattacharyya S, Morrison PD, Fusar-Poli P, Martin-Santos R, Borgwardt S, Winton-Brown T, et al. Opposite effects of delta9-tetrahydrocannabinol and cannabidiol on human brain function and psychopathology. Neuropsychopharmacology. 2010;35:764-74.

8 Millar SA, Stone NL, Yates AS, O'Sullivan SE. A systematic review on the pharmacokinetics of cannabidiol in humans. Front Pharmacol. 2018;9:1365.

9 Birnbaum AK, Karanam A, Marino SE, Barkley CM, Remmel RP, Roslawski M, et al. Food effect on pharmacokinetics of cannabidiol oral capsules in adult patients with refractory epilepsy. Epilepsia. 2019;60:1586-92.

10 Wheless JW, Dlugos D, Miller I, Oh DA, Parikh N, Phillips S, et al. Pharmacokinetics and tolerability of multiple doses of pharmaceutical-grade synthetic cannabidiol in pediatric patients with treatmentresistant epilepsy. CNS Drugs. 2019;33:593-604.

11 Leber S, Heidenreich T, Stangier U, Hofmann SG. Processing of facial affect under social threat in socially anxious adults: mood matters. Depress Anxiety. 2009;26:196-206.

12 Rossi GN, Osório FL, Morgan CJ, Crippa JA, Bouso JC, Rocha JM, et al. The effects of Cannabidiol (CBD) and Delta-9-Tetrahydrocannabinol (THC) on the recognition of emotions in facial expressions: a systematic review of randomized controlled trials. Neurosci Biobehav Rev. 2020;118:236-46.

13 Zuardi AW, Guimarães FS, Moreira AC. Effect of cannabidiol on plasma prolactin, growth hormone and cortisol in human volunteers. Braz J Med Biol Res. 1993;26:213-7.

14 Zuardi AW, Rodrigues NP, Silva AL, Bernardo SA, Hallak JE, Guimarães FS, et al. Inverted U-shaped dose-response curve of the anxiolytic effect of cannabidiol during public speaking in real life. Front Pharmacol. 2017;8:259.

15 Lunn S, Diaz P, O'Hearn S, Cahill SP, Blake A, Narine K, et al. Human pharmacokinetic parameters of orally administered $\Delta^{9}$-tetrahydrocannabinol capsules are altered by fed versus fasted conditions and sex differences. Cannabis Cannabinoid Res. 2019;4: 255-64.

16 Rutter LA, Dodell-Feder D, Vahia IV, Forester BP, Ressler KJ, Wilmer JB, et al. Emotion sensitivity across the lifespan: mapping clinical risk periods to sensitivity to facial emotion intensity. J Exp Psychol Gen. 2019;148:1993-2005.

17 Norris $\mathrm{H}$. The action of sedatives on brain stem oculomotor systems in man. Neuropharmacology. 1971;10:181-91.

18 Zuardi AW, Cosme RA, Graeff FG, Guimarães FS. Effects of ipsapirone and cannabidiol on human experimental anxiety. J Psychopharmacol. 1993;7:82-8.
19 McNair DM, Frankenthaler LM, Czerlinsky T, White TW, Sasson S, Fisher S. Simulated public speaking as a model of clinical anxiety. Psychopharmacology (Berl). 1982;77:7-10.

20 Linares IM, Zuardi AW, Pereira LC, Queiroz RH, Mechoulam R, Guimarães FS, et al. Cannabidiol presents an inverted U-shaped dose-response curve in a simulated public speaking test. Braz $\mathrm{J}$ Psychiatry. 2019;41:9-14.

21 Arrais KC, Machado-de-Sousa JP, Trzesniak C, Santos Filho A Ferrari MC, Osório FL, et al. Social anxiety disorder women easily recognize fearfull, sad and happy faces: the influence of gender. J Psychiatr Res. 2010;44:535-40.

22 Ekman P, Friesen WV. Pictures of facial affect. Palo Alto: Consulting Psychologists; 1976.

23 Camargo SP, Queiroz RH, Malfara WR, Crippa JA, Zuardi AW, Hallak JE, et al. Development and validation of a liquid: liquid extraction and gas chromatography/mass espectrometry methodology for cannabidiol analysis in human plasma. Curr Pharm Anal. 2011;7:228-34

24 Arndt DL, de Wit H. Cannabidiol does not dampen responses to emotional stimuli in healthy adults. Cannabis Cannabinoid Res. 2017;2:105-13.

25 Winter H, Ginsberg A, Egizi E, Erondu N, Whitney K, Pauli E, et al. Effect of a high-calorie, high-fat meal on the bioavailability and pharmacokinetics of PA-824 in healthy adult subjects. Antimicrob Agents Chemother. 2013;57:5516-20.

26 Agurell S, Carlsson S, Lindgren JE, Ohlsson A, Gillespie H, Hollister L. Interactions of delta 1-tetrahydrocannabinol with cannabinol and cannabidiol following oral administration in man. Assay of cannabinol and cannabidiol by mass fragmentography. Experientia. 1981;37: 1090-2.

27 Ohlsson A, Lindgren JE, Andersson S, Agurell S, Gillespie H, Hollister LE. Single-dose kinetics of deuterium-labelled cannabidiol in man after smoking and intravenous administration. Biomed Environ Mass Spectrom. 1986;13:77-83.

28 Iffland K, Grotenhermen F. An update on safety and side effects of cannabidiol: a review of clinical data and relevant animal studies. Cannabis Cannabinoid Res. 2017;2:139-54.

29 Birnbaum AK, Karanam A, Marino SE, Barkley CM, Remmel RP, Roslawski M, et al. Food effect on pharmacokinetics of cannabidiol oral capsules in adult patients with refractory epilepsy. Epilepsia. 2019;60:1586-92.

30 Schier AR, Ribeiro NP, e Silva AC, Hallak JE, Crippa JA, Nardi AE, et al. Cannabidiol, a cannabis sativa constituent, as an anxiolytic drug. Braz J Psychiatry. 2012;34 Suppl 1:S104-10.

31 Dos Santos RG, Guimarães FS, Crippa JA, Hallak JE, Rossi GN, Rocha JM, et al. Serious adverse effects of cannabidiol (CBD): a review of randomized controlled trials. Expert Opin Drug Metab Toxicol. 2020;16:517-26.

32 de Faria SM, de Morais Fabrício D, Tumas V, Castro PC, Ponti MA, Hallak JE, et al. Effects of acute cannabidiol administration on anxiety and tremors induced by a Simulated Public Speaking Test in patients with Parkinson's disease. J Psychopharmacol. 2020;34:189-96. 